in some of the fatal cases restlessness and confusion were succeeded by coma and death. Some had loss of memory for the acute stage of the illness. There were also changes in peripheral sensation-hypersensitivity to pain and touch, " pins and needles," or a feeling of "lying on crumbs." Myelitis was recorded in one patient after the end of the acute stage of illness.

The patients developed a tendency to bleed, notably from the gums and from needle punctures. Haematemesis and melaena occurred, but the rash was never haemorrhagic. There was marked thrombocytopenia (less than 10,000 per cu. mm. in two fatal cases) but no other changes in bloodclotting factors sufficient to account for the bleeding. Transaminase levels (especially S.G.O.T.) were raised, and atypical lymphocytes and plasma cells were seen in association with leukopenia. Serum proteins (all components) declined in all cases, but significant proteinuria was not seen.

Seven patients died and the remainder had a slow convalescence. Three patients relapsed. ${ }^{4}$ Of these, one had a marked rise in serum transaminase 31 days after recovery; the second had a similar rise 73 days after, associated with an acute psychosis, and the virus was isolated from a liver biopsy; the third appears to have infected his wife by sexual intercourse 11 weeks after recovery, and the virus was recovered from his semen.

The laboratory workers acquired infection from contact with monkey tissues or cultures from them, and not from contact with or care of the intact infected animals. ${ }^{5}$ However, several cases were infected in hospital by contact with the blood of patients-a risk aggravated by their bleeding tendency. The agent was isolated from urine and throat washings of some patients as well as from blood. ${ }^{4}$

Necropsy examination indicated that necrotic changes had first affected the liver and lymphatic system, then the pancreas, gonads, adrenals, hypophysis, thyroid, kidneys, and skin. There was also evidence, supported by liver biopsy findings, that the liver damage was quickly repaired by regeneration after the acute stage. Basophilic bodies were seen in cells in and around necrotic lesions. In lymphoid tissue there was a notable transformation to plasma cells and monocyte-like cells. Similar cells had diffusely infiltrated the mucosa of stomach, small intestines, and less of the large intestine. All cases had severe parenchymal damage and evidence of tubular failure in the kidneys, associated with cerebral oedema. In patients who died in coma there was either some evidence of encephalitis (glial nodules) or a haemorrhagic state in the central nervous system. ${ }^{7}$

Some laboratory studies are also reported on the isolation of the virus in guinea-pigs and monkeys ${ }^{8}$; and serological studies, including fluorescent antibody work, ${ }^{9}$ gave similar findings to those obtained at the Microbiological Research Establishment, Porton, ${ }^{10}{ }^{11}$ showing that the agent obtained there and in Germany is identical. Growth of the agent in human amnion cells is reported, and electronmicroscopy of it in blood gave a length of $800-1,000 \mathrm{~m} \mu .{ }^{12}{ }^{13}$

The accumulated evidence shows that this is a previously unrecognized disease caused by an agent probably new to medical science. As the origin of the infection remains obscure, it would be unwise to assume that infections of man will not recur. Moreover they may come next time from another source, for vervet monkeys are clearly not the maintenance hosts of the virus. For instance, it is infective also for rhesus monkeys. The cases reported here were apparently due to contact with blood or tissues, but the presence of the agent in the throat and urine of both man and monkeys during the disease, and transmission of it by sexual intercourse, suggest that the epidemiological propensities of the infection may be wide. In any case of obscure febrile illness the attending doctor should always ensure that he has made inquiries about contact with wild or pet animals. Though the infection has not yet been found in monkeys in a latent or non-fatal form, the possibility cannot be dismissed that it will be either in them or in other species of animals in view of its persistence in man for up to 11 weeks after recovery. No specific treatment has been found for this disease.

\section{Cystic Fibrosis and Fertility}

More and more children with the autosomal recessive condition cystic fibrosis of the pancreas are surviving into adult life and eventually will be marrying. The genetic risks to their children are not high. Such survivors will transmit the gene to all their children, but the children cannot be homozygotes unless the other parent is also a carrier. In countries where the incidence of the disease is about 1 in 2,000 the carrier rate will be between 1 in 20 and 1 in 25 and the risk to children of those affected between 1 in 40 and 1 in 50 . Detection of carriers is probably not too far away, and this will provide a further safeguard.

Girl survivors are known to be fertile; already at least 11 liveborn children have been reported and these children have all been unaffected. ${ }^{1}$ So far, however, no surviving males have had children, and C. R. Denning, S. C. Sommers, and H. J. Quigley ${ }^{2}$ have recently reported that 8 adult male patients with only mild pulmonary disease all had complete azoospermia. Not even any dead or malformed spermatozoa were found on careful search. The volume of semen was small and its turbidity was increased, but viscosity was normal and fructose was present. Testicular biopsy in one patient and necropsy of nine other male patients who died of cystic fibrosis showed spermatogenesis occurring and normal prostate and seminal vesicles.

The cause of the azoospermia is not established. The authors note that many spermatocytes were binucleate, or contained cytoplasmic inclusions or showed incomplete meiosis. They postulated some genetic abnormality of spermatogenesis. They also found low serum levels of vitamin $\mathrm{A}$ and vitamin $\mathrm{E}$ in the patients and suggested that this might be relevant. On the analogy of the pancreatic and salivary lesions the most probable mechanism of the azoospermia, however, is that there is a defect in transport of the spermatozoa owing to diminished water and electrolyte secretion.

1 Grand, R. J., Talamo, R. C., di Sant'Agnese, P. A., and Schwartz, R. H., f. Amer. med. Ass., 1966, 195, 993.

2 Denning, C. R., Sommers, S. C., and Quigley, H. J., Pediatrics, 1968, 41, 7 . 